\title{
Improving Adherence to Reach Out and Read: A Bookmark Intervention
}

\author{
Elsia A. Obus ${ }^{2}$, Natalie H. Brito ${ }^{2}$, Lauren Sanlorenzo ${ }^{2}$, Corinna Rea ${ }^{2}$, Laura Engelhardt ${ }^{2}$, \& Kimberly G. Noble ${ }^{1 *}$ \\ 'Teachers College, Columbia University, USA \\ ${ }^{2}$ Columbia University Medical Center, USA
}

\section{Article Info}

\section{Article Notes}

Received: October 03, 2017

Accepted: November 03, 2017

\section{${ }^{*}$ Correspondence:}

Dr. Kimberly G. Noble

Teachers College, Columbia University

525 West 120 th Street

New York, NY 10027, USA

212-678-3486, E-mail: kgn2106@tc.columbia.edu

(C) 2017 Noble KG. This article is distributed under the terms of the Creative Commons Attribution 4.0 International License.

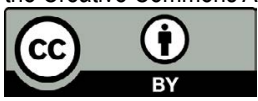

\section{Keywords}

Reach out and read

Reading

Language

Literacy

Intervention

Adherence

Implementation

Bookmark

\begin{abstract}
This study examines how the addition of a modest addendum to the well-established pediatric primary care program, Reach Out and Read (ROR), is associated with increased clinician adherence to ROR and caregiver home literacy behavior. This study took place in four ambulatory care clinics at a large urban medical center. All clinics received standard ROR training. Two of the four clinics received additional ROR training and bookmarks with agespecific advice about reading aloud with children. Following the intervention, medical providers reported no behavioral differences, however caregivers in the intervention group reported: more frequent trips to the library, receiving more books from their pediatrician, and receiving more advice on how to read with their child than caregivers in from the comparison clinics. Thus the addition of a modest training and bookmark intervention to the ROR program was associated with caregiver report of both increased clinician adherence to ROR and increased caregiver literacy behavior. The bookmark intervention may be an inexpensive way to improve the effects of the ROR program.
\end{abstract}

\section{Introduction}

Socioeconomic status (SES) is strongly associated with children's academic achievement and language development ${ }^{1}$, with disparities in language abilities appearing as early as 15 months $^{2}$ and persisting throughout the lifespan ${ }^{3}$. The home literacy environment, including caregiver literacy behaviors and availability of books and reading materials, may be a mediator through which SES affects children's language development ${ }^{4-6}$.

A number of interventions have aimed to improve home literacy environments of economically disadvantaged families and assist parents in engaging in reading activities with their children ${ }^{7-9}$, but many of these programs involve home-visiting, which can be labor-intensive and expensive, or in-school programs, which are not available in early childhood. The pediatric primary care setting, however, is a potentially effective, convenient, and inexpensive platform through which to implement early childhood intervention ${ }^{10,11}$. Eighty-six percent of children under five years old have seen a health care professional in the last six months ${ }^{12}$. Thus, pediatric clinics offer a location to which children and parents are coming often and early in life.

The current intervention is an extension of the well-established pediatric primary care program, Reach Out and Read (ROR) ${ }^{13}$. The ROR program has three main components: 1) literacy-rich waiting rooms with volunteers who read aloud to children, thereby modeling 
effective read-aloud strategies for parents, 2) anticipatory guidance about reading aloud given by clinicians to parents during the visit, and 3) the gift of an age-appropriate picture book to each child between the ages of 6 months and 5 years during the course of every well-visit ${ }^{14}$. The ROR program has over 5,800 program sites in all 50 states in the United States and is estimated to serve 4.7 million children annually ${ }^{15}$.

ROR has had great success at increasing enjoyment of and participation in child-centered literacy activities at home $\mathrm{e}^{16,17}$ and improving child vocabulary outcomes ${ }^{17,18}$. However, recent studies have suggested that the fidelity of implementation of the ROR program between clinics is quite variant. One study found that clinics that were rated by their ROR Regional Coalition Coordinator as being "very successful" gave out books to children at 87-99\% of wellchild visits, while clinics that were rated as "struggling" gave out books at only $2-54 \%$ of well-child visits ${ }^{19}$. A meta-analysis of the methodological quality of ROR found that only $9 \%$ of studies included fidelity measures of adherence to ROR guidelines and suggested that clinics had inconsistent book distribution and provision of anticipatory guidance ${ }^{20}$.

The current study examines the effects of an addendum to the ROR intervention, developed to improve adherence. All clinics in this study were already participating in the ROR program. At half of the clinics, providers received an additional training outlining the importance of ROR, its evidence base, and how to provide developmentally appropriate anticipatory guidance about home literacy practices to caregivers. Providers at these intervention clinics were also instructed to give out informational bookmarks with the ROR books, which included tips for reading with children of different ages. Previous research has shown that simple changes to the ROR protocol, like moving the location of books, can significantly improve clinician adherence to ROR and increase book distribution ${ }^{21}$. Additionally, caregivers who report receiving more ROR books also report engaging in more literacy activities with their children ${ }^{22}$. Thus it was hypothesized that the intervention clinics would more consistently give out ROR books and provide anticipatory guidance, and that the bookmark intervention would increase home literacy activities.

\section{Methods}

\section{Samples}

Convenience samples were recruited from four pediatric clinics located in the Harlem and Washington Heights neighborhoods of New York City. These clinics are part of the Columbia University Medical Center Ambulatory Care Network, and provide healthcare to predominantly lowincome families in the surrounding community.
Primary caregivers of pediatric patients between the ages of six months to five years were recruited from waiting rooms to complete a questionnaire regarding their demographic information and literacy-promoting behaviors. In addition to caregivers, primary care providers - including residents, attendings, and nurse practitioners - were recruited to complete a questionnaire regarding their application of the ROR program. Caregivers and primary care providers were recruited before and after the intervention; thus different groups of caregivers and primary care providers completed the questionnaires across time points.

\section{Procedures}

Implementation of the standard ROR program was already in place at the time of the study. Clinics distributed age-appropriate books to children during routine wellchild checks. Once a year, residents received a lecture about ROR, but providers received no additional training. Two of the four clinics were designated as intervention sites, while the remaining two clinics served comparison sites.

\section{Pre-Intervention}

Primary caregivers were approached in the pediatric clinic waiting rooms and invited to complete a questionnaire regarding basic demographic characteristics, the home reading environment and literacy-based beliefs and practices. Questionnaires were administered orally in English and Spanish by a trained experimenter.

Clinicians at the four pediatric clinics were emailed a unique link to a 12-item questionnaire that addressed how often the providers remembered to distribute books; how often they gave counseling and modeled appropriate reading behavior; and how familiar they were with the ROR program and its application.

\section{Intervention}

All clinicians at the intervention clinics participated in a training session in which they learned about the components of ROR, its evidence base, and how to administer developmentally appropriate guidance to support families' literacy efforts. Providers were also instructed to give families ROR-approved bookmarks, designed by the investigators, along with their free book. The bookmarks included simple steps for engaging children in reading activities and encouraging literacy across development (Appendix A). Six developmentally appropriate bookmarks were designed to target children at different ages, and all bookmarks were printed in English and Spanish. The bookmarks also served to prompt physicians to counsel families on reading behaviors during well-visits and were stocked near the ROR books in all examination rooms at the intervention sites. 


\section{Post-Intervention}

Providers at the four pediatric clinics were emailed links to the same 12-item questionnaire completed before the intervention. Primary caregivers were again approached in each clinic's pediatric waiting rooms and invited to complete the same questionnaire that had been administered before the intervention. Time between preand post-intervention questionnaire was approximately 9-months. The intervention was implemented for the entire duration between the administration of the pre- and post-intervention questionnaires.

\section{Analysis Plan}

In the caregiver analyses, between-subjects t-tests were used to examine any differences in demographic factors (child age, caregiver age, caregiver years of education, or family income-to-needs) between the intervention and comparison caregivers, both before and after the intervention. Chi-squared tests were used to investigate whether caregiver responses regarding reading-related activities in the home differed in the intervention vs. comparison groups, both prior to and after the intervention. For the provider analyses, chisquared tests were used to examine differences across clinician position type (attending, resident, or nurse practitioner), as well as differences between groups on reported rates of formal ROR training. For between-group analyses of providers post-intervention, the mean of the pre-intervention responses was used as a control. Because surveys of caregivers were anonymous, we cannot know the percentage of caregivers who answered both the preintervention and post-intervention surveys; we therefore did not control for mean pre-intervention behaviors when analyzing between-group post-intervention differences.

\section{Results}

\section{Caregivers}

Caregivers completed a total of 180 questionnaires before the intervention ( 90 from intervention clinics, 90 from comparison clinics) and 180 questionnaires after the intervention (90 from intervention clinics, 90 from comparison clinics). Child and caregiver demographic characteristics are shown in Table 1. Because the caregivers were not necessarily the same in the pre- and post-

Table 1. Child and caregiver demographic characteristics pre- and post-intervention.

\begin{tabular}{|c|c|c|c|c|}
\hline \multirow{2}{*}{ Demographic Characteristics } & \multicolumn{2}{|c|}{ Comparison Group } & \multicolumn{2}{|c|}{ Intervention Group } \\
\hline & Pre & Post & Pre & Post \\
\hline Child Age, mean (SD), mo & $34.16(18.25)$ & $33.85(17.22)$ & $30.25(16.40)$ & $37.99(18.45)$ \\
\hline \multicolumn{5}{|l|}{ Child Sex } \\
\hline Male & $51(57 \%)$ & $37(41 \%)$ & $39(43 \%)$ & $45(50 \%)$ \\
\hline Female & $39(43 \%)$ & $53(59 \%)$ & $51(57 \%)$ & $45(50 \%)$ \\
\hline \multicolumn{5}{|l|}{ Child Ethnicity } \\
\hline Non-Hispanic White & $0(0 \%)$ & $0(0 \%)$ & $0(0 \%)$ & $2(2 \%)$ \\
\hline Hispanic & $61(68 \%)$ & $66(73 \%)$ & $86(96 \%)$ & $81(90 \%)$ \\
\hline African-American & $20(22 \%)$ & $13(14 \%)$ & $1(1 \%)$ & $2(2 \%)$ \\
\hline Other & $9(10 \%)$ & $11(12 \%)$ & $3(3 \%)$ & $5(6 \%)$ \\
\hline \multicolumn{5}{|l|}{ Child Language } \\
\hline English & $37(41 \%)$ & $31(35 \%)$ & $26(29 \%)$ & $13(14 \%)$ \\
\hline Spanish & $34(38 \%)$ & $38(42 \%)$ & $44(49 \%)$ & $52(58 \%)$ \\
\hline Both & $12(13 \%)$ & $11(12 \%)$ & $19(21 \%)$ & $22(24 \%)$ \\
\hline Other & $7(8 \%)$ & $10(11 \%)$ & $1(1 \%)$ & $3(3 \%)$ \\
\hline \multicolumn{5}{|l|}{ Gestational Age at Birth } \\
\hline Less than 35 weeks & $13(14 \%)$ & $5(6 \%)$ & $4(4 \%)$ & $10(11 \%)$ \\
\hline 35-37 weeks & $14(16 \%)$ & $11(12 \%)$ & $13(14 \%)$ & $6(7 \%)$ \\
\hline Over 37 weeks & $72(80 \%)$ & $74(82 \%)$ & $62(69 \%)$ & $74(82 \%)$ \\
\hline \multicolumn{5}{|l|}{ Family Members in the Home } \\
\hline \# of Adults, mean (SD) & $2.19(0.87)$ & $2.07(0.87)$ & $2.08(0.78)$ & $2.11(1.31)$ \\
\hline \# of Children, mean (SD) & $1.93(1.09)$ & $1.84(1.09)$ & $1.89(1.05)$ & $1.73(1.01)$ \\
\hline \multicolumn{5}{|l|}{ Parents Married } \\
\hline Yes & $33(37 \%)$ & $27(30 \%)$ & $27(30 \%)$ & $39(43 \%)$ \\
\hline No & $56(62 \%)$ & $63(70 \%)$ & $63(70 \%)$ & $51(57 \%)$ \\
\hline Caregiver Education, mean (SD), years & $11.96(2.82)$ & $11.76(2.49)$ & $12.36(2.35)$ & $12.03(2.70)$ \\
\hline Family Income, mean (SD) & $\begin{array}{c}22,012 \\
(15,309)\end{array}$ & $\begin{array}{c}18,239 \\
(12,736)\end{array}$ & $\begin{array}{c}27,236 \\
(14,189)\end{array}$ & $\begin{array}{c}20,000 \\
(13,651)\end{array}$ \\
\hline Family Income to Needs, mean (SD) & $0.94(0.68)$ & $0.78(0.52)$ & $1.17(0.65)$ & $0.87(0.60)$ \\
\hline
\end{tabular}


intervention groups, we carefully compared demographics across the intervention and comparison groups, both before and after implementation of the intervention. There were no differences in child age, caregiver age, caregiver years of education, or family income-to-needs between the groups before or after the intervention ( $p$ 's $>0.05$ ). Additionally, there were no differences in caregiver responses regarding reading-related activities in the home between groups before the intervention ( $p$ 's > 0.08). Therefore, only postintervention responses were analyzed.

Following the intervention, caregivers in the intervention group reported more frequent trips to the library, $X^{2}(3,92)=10.71, p=0.01$; receiving more books from their pediatrician, $X^{2}(2,180)=9.51, p=0.01$; and receiving more reading advice on how to read with their child, $X^{2}(4,180)=16.82, p<0.01$ (Figure 1). In contrast, the comparison group reported reading with their child in the previous 24 hours more often than the intervention group,
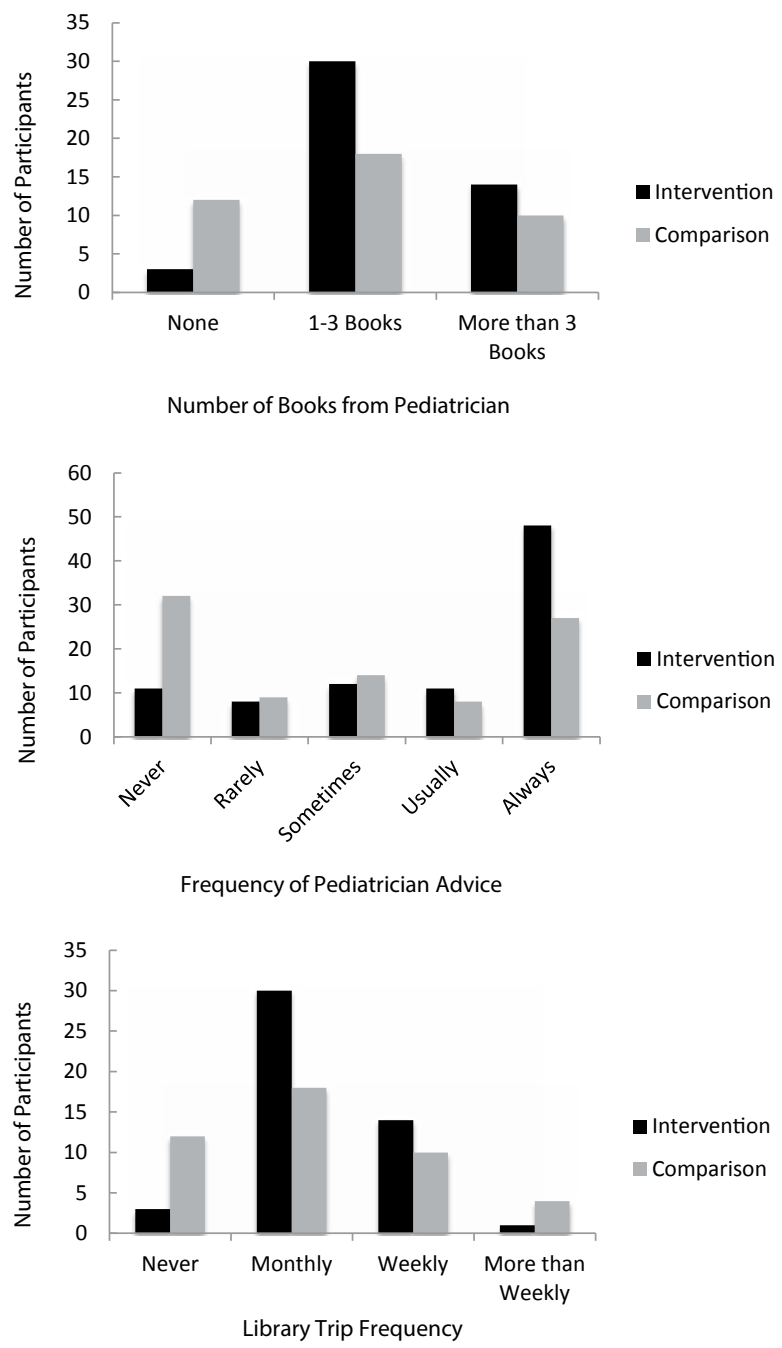

Figure 1: Caregiver responses from intervention and comparison clinics about books received, advice received, and library trip frequency.
$X^{2}(1,180)=4.58, p=0.03$. Caregiver responses by group are shown in Table 2 . No other significant differences were found between caregiver groups.

\section{Providers}

Providers completed a total of 59 questionnaires before the intervention (31 from intervention clinics, 29 from comparison clinics) and 51 questionnaires after the intervention (24 from intervention clinics, 27 from comparison clinics). The clinician position type (attending, resident, or nurse practitioner) did not differ significantly across intervention and comparison clinics before or after the intervention. There were no differences between groups on reported rates of formal ROR training, though the intervention group was more likely to report having received training on counseling families about reading, $X^{2}$ $(1,59)=6.11, p=0.01$.

The same clinicians did not complete both pre- and post-intervention questionnaires, and therefore a repeated measures type analysis was not feasible. In order to control for pre-intervention behaviors, responses on pre-intervention questionnaires were controlled for in subsequent analyses by taking the difference between the post-intervention responses of each individual and the mean of pre-intervention responses by each clinic. In this way, pre-intervention responses were treated as a baseline for each clinic, and post-intervention responses reflected increases or decreases from the average clinic response. The also made the dependent variables more normally distributed, allowing for parametric analyses.

Following the intervention, no differences were found between the intervention and comparison clinics on book distribution behavior, reading counseling behavior, comfort in giving advice, or frequency in modeling reading techniques. Provider responses are shown in Table 3. In addition to possible changes in behavior, qualitative questions were asked about the clinicians' experiences in promoting literacy with their patients. Both before and after the intervention, clinicians reported no differences in time of book distribution (beginning, middle, or end of well-visit) and that there was usually an age-appropriate book available in the exam room. Additionally, providers reported that the biggest barrier to implementing ROR was not having enough time during the well-visit. These qualitative results did not differ significantly by intervention group.

\section{Discussion}

In line with our hypothesis, we found that caregivers in the intervention clinics reported receiving more books from their pediatricians than caregivers at non-intervention clinics. We believe that the additional ROR training that the clinicians at these clinics received increased the number of 
Table 2. Caregiver responses to questions regarding ROR experience and literacy activities.

\begin{tabular}{|c|c|c|c|c|c|}
\hline & & $\begin{array}{l}\text { Comparison } \\
\text { Group (N) }\end{array}$ & $\begin{array}{l}\text { Intervention } \\
\text { Group (N) }\end{array}$ & $x^{2}$ & $\mathrm{p}$ \\
\hline \multirow{3}{*}{$\begin{array}{l}\text { How many books have you received from your pediatri- } \\
\text { cian? }\end{array}$} & None & 10 & 8 & \multirow{3}{*}{9.51} & \multirow{3}{*}{0.01} \\
\hline & 1-3 books & 32 & 15 & & \\
\hline & More than 3 books & 48 & 67 & & \\
\hline \multirow{5}{*}{$\begin{array}{l}\text { How often did your pediatrician counsel you about how } \\
\text { to read with your child at well-child visits after your child } \\
\text { turned } 6 \text { months? }\end{array}$} & Never & 32 & 11 & \multirow{5}{*}{16.82} & \multirow{5}{*}{$<0.01$} \\
\hline & Rarely & 9 & 8 & & \\
\hline & Sometimes & 14 & 12 & & \\
\hline & Usually & 8 & 11 & & \\
\hline & Always & 27 & 48 & & \\
\hline \multirow{5}{*}{$\begin{array}{l}\text { If you have a library card, how often do you go to the } \\
\text { library on average? }\end{array}$} & Do not have a library card & 46 & 42 & \multirow{5}{*}{0.71} & \multirow{5}{*}{0.01} \\
\hline & Never & 12 & 3 & & \\
\hline & Once a month & 18 & 30 & & \\
\hline & Once a week & 10 & 14 & & \\
\hline & More than once a week & 4 & 1 & & \\
\hline \multirow{2}{*}{$\begin{array}{l}\text { Have you and your child read together during the previ- } \\
\text { ous } 24 \text { hours? }\end{array}$} & Yes & 62 & 48 & \multirow{2}{*}{4.58} & \multirow{2}{*}{0.03} \\
\hline & No & 28 & 42 & & \\
\hline
\end{tabular}

Table 3. Provider responses to questions regarding ROR adherence and comfort.

\begin{tabular}{|c|c|c|c|}
\hline & & $\begin{array}{l}\text { Comparison } \\
\text { Group (N) }\end{array}$ & $\begin{array}{l}\text { Intervention } \\
\text { Group (N) }\end{array}$ \\
\hline \multirow{5}{*}{$\begin{array}{l}\text { How often do you remember to distribute Reach Out and } \\
\text { Read Books at your } \\
\text { well-child visits? }\end{array}$} & Never & 0 & 0 \\
\hline & Rarely & 0 & 0 \\
\hline & Sometimes & 9 & 2 \\
\hline & Usually & 36 & 21 \\
\hline & Always & 11 & 31 \\
\hline \multirow{5}{*}{$\begin{array}{l}\text { How often do you give parents developmentally appropri- } \\
\text { ate counseling regarding reading? }\end{array}$} & Never & 0 & 0 \\
\hline & Rarely & 10 & 3 \\
\hline & Sometimes & 30 & 17 \\
\hline & Usually & 14 & 25 \\
\hline & Always & 2 & 9 \\
\hline \multirow{5}{*}{$\begin{array}{l}\text { How comfortable do you feel giving parents developmen- } \\
\text { tally appropriate counseling regarding reading? }\end{array}$} & Uncomfortable & 3 & 5 \\
\hline & Somewhat uncomfortable & 12 & 1 \\
\hline & Somewhat comfortable & 25 & 18 \\
\hline & Mostly comfortable & 12 & 22 \\
\hline & Completely comfortable & 4 & 8 \\
\hline \multirow{5}{*}{ How often do you model reading techniques with families? } & Never & 19 & 3 \\
\hline & Rarely & 18 & 10 \\
\hline & Sometimes & 15 & 31 \\
\hline & Usually & 4 & 8 \\
\hline & Always & 0 & 2 \\
\hline
\end{tabular}

Note: All provider results were not significant.

well-visits at which they gave out a book. It is possible that the training made the ROR program a more salient aspect of their job at the clinic and therefore they remembered to give a book as part of every well-child visit. It is also possible that because the training included background on the scientific evidence for ROR, it increased clinician buy-in to the program, which in turn increased adherence.

It is interesting to note that, although parents in the intervention group reported receiving books more frequently, no significant differences were found following the intervention in clinician-report of book distribution, although trends suggested that clinicians in intervention clinics reported more compliance with ROR protocols. Previous studies have also reported discrepancies between caregiver-report, clinician-report, and clinic-report of the number of books that families have received ${ }^{23}$. One possible interpretation is that parents may provide less reliable reports than providers. However, we note that caregivers 
were asked to quantify the actual number of books received from their pediatrician, whereas clinicians were asked, "How often do you remember to distribute Reach Out and Read books at your well-child visits between 6 months and 5 years?" with possible responses being a Likert-type scale ranging from "Always" to "Never." It is therefore possible that, because parents were asked to reporta specific number of books, their reports may have been more accurate than the qualitative estimates of the providers. The memory for particular books may be more tangible than the estimate of how often books were distributed. In many ROR clinics, clinicians include whether a book was given in the official medical record. Future studies should consider collecting medical records, in addition to parent and clinician report, to more accurately track book distribution.

In addition to reporting having received more books, caregiver in the intervention clinics reported receiving more counseling about reading with their child from the pediatrician than caregivers at the comparison clinics. The bookmarks themselves had information about how to read with children of different ages. It is possible that the bookmarks served as physical reminders to the clinicians to provide anticipatory guidance and perhaps even as aids to clinicians who did not remember age-appropriate tips for reading. It is also possible that both the additional training and the bookmark together improved anticipatory guidance outcomes and future research is needed to parse out their independent effects.

Finally, caregivers at intervention clinics reported more frequent trips to the library than caregivers at control clinics. This result may be the most notable as it relates directly the child's literacy environment. It indicates that increased book distribution and anticipatory guidance may positively affect the literacy-related activities that caregivers engage in with their children.

These preliminary results suggest that the addition of the bookmark intervention to the established ROR program may increase clinician adherence to ROR, which in turn may increase caregivers' participation in literacy-related activities. However, the study has several limitations. It was not longitudinal within individuals, which limits our ability to draw conclusions about the causative associations amongst the bookmark intervention, adherence to ROR protocols, and home literacy outcomes. Additionally, we collected post-intervention data at only one time point, 9 months after the implemented of the intervention, therefore we were unable to test how soon after implementation increased adherence and home literacy behaviors began. Future studies should seek to determine how quickly the bookmark intervention improves these outcomes. Furthermore, our data consist entirely of self-report measures. The use of more objective data sources in future studies, such as clinical records of book distribution, could improve reliability and validity. Finally, the small number of clinics limits the generalizability of our results. In the future we hope to study the effects of the bookmark addendum to ROR on a larger scale. Despite these limitations, our results suggest that the addition of an inexpensive add-on to the typical ROR program - in the form of an informational bookmark for parents and brief additional training for providers - could have positive impacts on clinician and caregiver literacy behavior.

\section{Acknowledgements}

We gratefully acknowledge funding by the John M. Driscoll Children's Fund (to KGN), the Columbia University Dept. of Pediatrics, the GH Sergievsky Center, and Teachers College, Columbia University.

\section{Conflict of Interest statement}

The authors have no potential conflicts of interest and no corporate sponsers.

\section{References}

1. Sirin SR. Socioceconomic status and academic achievement: A metaanalytic review of research. Rev Educ Res. 2005; 75(3): 417-453.

2. Noble KG, Engelhardt LE, Brito NH, et al. Socioeconomic disparities in neurocognitive development in the first two years of life. Dev Psychobiol. 2015; 57(5): 535-551.

3. Bradley RH, Corwyn RF. Socioeconomic status and child development. Annu Rev Psychol. 2002; 53(1): 371-399.

4. Burgess SR, Hecht SA, Lonigan CJ. Relations of the home literacy environment (HLE) to the development of reading-related abilities: A one-year longitudinal study. Read Res Q. 2002; 37(4): 408-426.

5. Payne AC, Whitehurst GJ, Angell AL. The role of the home literacy environment in the development of language ability in preschool children from low-income families. Early Child Res Q. 1994; 9(3-4): 427-440.

6. Weigel DJ, Martin SS, Bennett KK. Contributions of the home literacy environment to preschool-aged children's emerging literacy and language skills. Early Child Dev Care. 2006; 176(3-4): 357-378.

7. Landry SH, Smith KE, Swank PR, et al. The effects of a responsive parenting intervention on parent-child interactions during shared book reading. Dev Psychol. 2012: 48(4): 969-986.

8. Leffel K, Suskind D. Parent-directed approaches to enrich the early language environments of children living in poverty. Semin Speech Lang. 2013; 34(4): 267-278.

9. Sim SSH, Berthelsen D, Walker S, et al. A shared reading intervention with parents to enhance young children's early literacy skills. Early Child Dev Care. 2014; 184(11): 1531-1549.

10. Shah R, Kennedy S, Clark MD, et al. Primary care-based interventions to promote positive parenting behaviors: A meta-analysis. Pediatrics. 2016; 137(5): e20153393.

11. Cates CB, Weisleder A, Mendelsohn AL. Mitigating the effects of family poverty on early child development through parenting interventions in primary care. Acad Pediatr. 2016; 16(3): S112-S120.

12. National Center for Health Statistics. Summary health statistics: National health interview survey. Hyattsville, Maryland. 2014.

13. Needlman R, Klass P, Zuckerman B. Reach out and get your patients to read. Contemp Pediatr. 2002; 19(1): 51-69. 
14. Klass P, Dreyer BP, Mendelsohn AL. Reach out and read: Literacy promotion in pediatric primary care. Adv Pediatr. 2009; 56: 11-27.

15. Reach Out \& Read. Reach Out \& Read website. http://www. reachoutandread.org. Accessed June 22, 2017.

16. Golova N, Alario AJ, Vivier PM, et al. Literacy promotion for Hispanic families in a primary care setting: A randomized, controlled trial. Pediatrics. 1999; 103(5): 993-997.

17. High PC, LaGasse L, Becker S, et al. Literacy promotion in primary care pediatrics: Can we make a difference. Pediatrics. 2000; 105: 927-934.

18. Mendelsohn AL, Mogilner LN, Dreyer BP, et al. The impact of clinicbased literacy intervention on language development in inner-city preschool children. Pediatrics. 2001; 107(1): 130-134.

19. King TM, Muzaffar S, George M. The role of clinic culture in implementation of primary care interventions: The case of reach out and read. Acad Pediatr. 2009; 9(1): 40-46.

20. Pelatti CY, Pentimonti JM, Justice LM. Methodological review of the quality of reach out and read: Does it "work". Clin Pediatr. 2014; 53(4): 343-350.

21. Monge MC, Trinh JV, Woods SK. Improving med-peds resident compliance with reach out and read book distribution. Acad Pediatr. 2011; 11(4): e12-e12.

22. Rikin S, Glatt K, Simpson P, et al. Factors associated with increased reading frequency in children exposed to reach out and read. Acad Pediatr. 2015; 15(6): 651-657.

23. Weitzman CC, Roy L, Walls T, et al. More evidence for reach out and read: A home-based study. Pediatrics. 2004; 113(5): 1248-1253. 